\title{
OPTIMISING CLASSIFIERS FOR THE DETECTION OF PHYSIOLOGICAL DETERIORATION IN PATIENT VITAL-SIGN DATA
}

\author{
Sara Khalid, David A. Clifton, Lei Clifton and Lionel Tarassenko \\ Institute of Biomedical Engineering, Dept. of Engineering Science, University of Oxford \\ Old Road Campus, Roosevelt Drive, Oxford, OX3 7DQ, U.K. \\ \{sara.khalid, david.clifton, lei.clifton, lionel.tarassenko\}@eng.ox.ac.uk
}

Keywords: Novelty detection, Multi-class classification, SVM, MLP.

\begin{abstract}
Hospital patient outcomes can be improved by the early identification of physiological deterioration. Automatic methods of detecting patient deterioration in vital-sign data typically attempt to identify deviations from assumed "normal" physiological condition. This paper investigates the use of a multi-class approach, in which "abnormal" physiology is modelled explicitly. The success of such a method relies on the accuracy of data annotations provided by clinical experts. We propose an approach to estimate class labels provided by clinicians, and refine those labels such they may be used to optimise a multi-class classifier for identifying patient deterioration. We demonstrate the effectiveness of the proposed methods using a large data-set acquired in a 24-bed step-down unit.
\end{abstract}

\section{INTRODUCTION}

Adverse events in patient condition are often preceded by physiological deterioration evident in vitalsign data (Buist et al., 1999), and it is wellunderstood that patient outcomes can be improved by detecting this deterioration sufficiently early (NPSA, 2007). Machine learning techniques have been shown to be able to detect such physiological deterioration by analysing vital-sign data acquired from patient monitors connected to acutely ill hospital patients, such as Parzen window estimators (Tarassenko, 2005).

A large number of manual methods (Smith, 2008) have been developed to allow clinicians to identify patient deterioration on the general ward, based on periodically-collected vital sign data, typically acquired every two to four hours.

Both manual and automatic methods typically perform novelty detection (or one-class classification), in which deviations from some assumed "normal" behaviour are identified. This is a common approach to the condition monitoring of critical systems (Tarassenko, 2009), for which large numbers of examples of "normality" exist, but where there are comparatively too few examples of system failure to construct a multi-class classifier, in which known failure conditions are explicitly modelled.

However, should sufficient examples of patient deterioration be available, a multi-class approach may be taken. It is assumed a priori that, given sufficient examples of system failure, a multi-class classifier will outperform a one-class classifier due to the inclusion of more information in the classifier (Bishop, 2006). This paper describes an investigation in which a large data-set of patient vital-sign data was acquired during a clinical study such that a multi-class approach to identifying patient deterioration may be taken. For this to be successful, accurate class labels for the data are required. We investigate the reliability of class labels provided by clinicians, and propose methods to (i) estimate and refine those labels automatically, and (ii) use the resultant labels to optimise a multi-class classifier for detecting patient deterioration.

\section{ESTIMATING CLINICAL LABELS}

Obtaining accurate class labels for large, multivariate data-sets of vital signs is particularly difficult. The data-set considered in the investigation described by this paper, for example, was four- 
dimensional, comprising heart rate (HR), breathing rate (BR), peripheral arterial oxygen saturation $\left(\mathrm{SpO}_{2}\right)$, and the arithmetic mean of systolic and diastolic blood pressures (the systolic-diastolic average, or SDA), was acquired from 332 patients in a step-down unit, and contains over 18,000 hours of patient data (Tarassenko, 2005). It is impractical for clinical experts to annotate such a large data-set in its entirety.

The approach taken with the data-set was to determine retrospectively which periods of patient data exceeded standard "medical emergency team" (MET) criteria (Smith, 2008). The latter are standard thresholds on each vital sign that, if exceeded, should result in the clinical review of the patient. Periods of patient data that exceeded the MET criteria for at least four minutes were shown to a panel of clinicians, who then determined which periods were due to artefact (such as a sensor becoming detached from the patient), and which were sufficiently abnormal to require patient review. We here term the latter class labels of "abnormal" patient condition $C_{2}$, and will refer to examples of "normal" patient condition to have class label $C_{1}$.

As vital signs can take more extreme values during periods of abnormal physiology, the distributions of data from periods labelled $C_{2}$ have heavier tails than the distributions of data from "normal" patients. However, there is significant overlap between the distributions of data from the two classes. Additionally some types of physiological abnormality are not represented in the data-set as frequently as other types; e.g., apnoea and bradycardia (low BR and HR, respectively) are under-represented in comparison with tachypnoea and tachycardia (high BR and HR, respectively). We found that this imbalance leads to a linear classifier trained using such data successfully classifying the majority of the more well-represented tachypnoea and tachycardia data, while misclassifying the under-represented apnoea and bradycardia data.

Similarly, a non-linear classifier trained using the original labels incorrectly includes the distribution of bradycardia and apnoea data within its decision boundary, and hence misclassifies test data from this region of data space as belonging to class $C_{1}$.

The remainder of this paper investigates methods for refining class labels $C_{1}$ and $C_{2}$, such that they may be used to construct a multi-class classifier that successfully classifies under-represented types of "abnormal" data. We will illustrate the procedure using bivariate analysis, such that the decision boundary of a classifier may be examined. The application to the full multivariate data-set (e.g., 4- dimensional in this example) is considered in Section 4.

\section{REFINING CLINICAL LABELS TO OPTIMISE THE CLASSIFIER}

The left-hand plot of Figure 1 shows all of the data from the two classes in the bivariate space of HR and BR. Clusters of $C_{2}$ data corresponding to apnoea, tachypnoea, bradycardia, and tachycardia may be seen in the figure, although data from class $C_{1}$ often overlap with those clusters. Intuitively, we wish to increase the separation between the two classes such that a classifier trained using those labels results in a decision boundary that correctly classifies data from all modes of class $C_{2}$. We propose a method for doing so using an estimate of the probability density function (pdf) of the entire dataset.

\subsection{Defining a Multivariate Distribution to Estimate Labels}

We approximated the pdf of the whole data-set using a Parzen windows estimator (Bishop, 2006), after reducing the size of the data-set to 400 prototype patterns using $k$-means clustering with $k=400$ cluster centres. The covariance $\sigma^{2}$ of the 400 kernels in the pdf was set using the heuristic proposed in (Bishop, 2006). Given some data-point $\mathbf{x}^{\prime}$, its density $\kappa_{\mathbf{x}}=p\left(\mathbf{x}^{\prime}\right)$ defines a contour on the pdf. We then define a probability $\mathrm{P}\left[\kappa_{\mathbf{x}},\right]$ as follows:

$$
\mathrm{P}\left[\kappa_{\mathbf{x}^{\prime}}\right]=\int_{\kappa_{\mathbf{x}^{\prime}}}^{\kappa_{\mathrm{m}}} p(\mathbf{x}) d \mathbf{x}
$$

where $\kappa_{\mathrm{m}}=\max [p]$, the density at the mode of the pdf, $p$. Thus $\mathrm{P}\left[\kappa_{\mathbf{x}}\right]$ is the probability mass contained by integrating the pdf from its highest point down to the probability density contour $\kappa_{\mathbf{x}}$ '. This represents the probability that some random data-point $\mathbf{x}$ distributed according to $p$ will take a density value higher than density value $\kappa_{\mathbf{x}}$; ; i.e., $\mathrm{P}\left[\kappa_{\mathbf{x}}{ }^{\prime}\right] \equiv \mathrm{P}[p(\mathbf{x}) \geq$ $\kappa_{\mathbf{x}}$ ']. Thus, as $\mathrm{x}^{\prime}$ varies throughout the data space, its probability density will vary over the range $\left[\begin{array}{ll}0 & \kappa_{\mathrm{m}}\end{array}\right]$, and thus $\mathrm{P}\left[\kappa_{\mathbf{x}^{\prime}}\right]$ will vary over the range [ $\left[\begin{array}{ll}0 & 1\end{array}\right]$ correspondingly.

We define a threshold $T$ on $\mathrm{P}\left[\kappa_{\mathbf{x}^{\prime}}\right]$, and consider which data have $\mathrm{P}\left[\kappa_{\mathbf{x}}{ }^{\prime}\right] \geq T$ for varying values of $T$.

As described above, we expect data that lie furthest from the mode of the distribution of the whole 

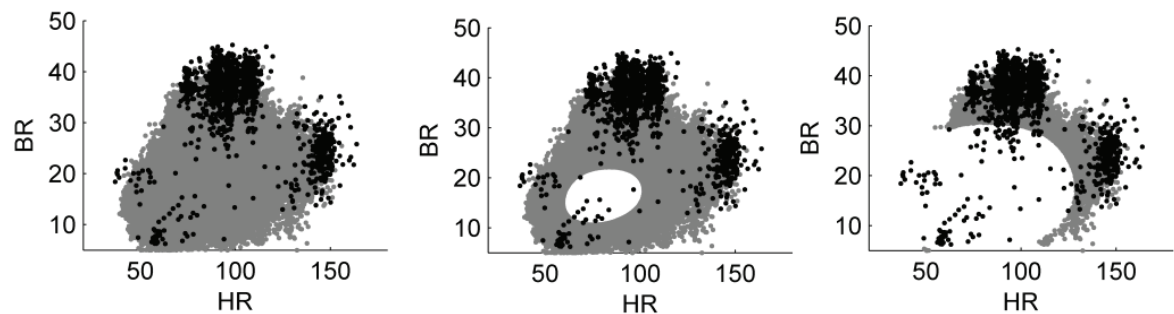

Figure 1: Data from classes $C_{1}$ and $C_{2}$ shown in grey and black, respectively. The left-hand plot shows all data from classes $C_{1}$ and $C_{2}$. Data x from $C_{1}$ are shown where $\mathrm{P}\left[\kappa_{\mathrm{x}}\right] \geq T$, for $T=0,0.5$, and 0.9 from left to right, respectively, as described in Section 3.1.

data-set to take largest value of $\mathrm{P}\left[\kappa_{\mathbf{x}}{ }^{\prime}\right]$, and hence as $T$ is increased, the proportion of data that have $\mathrm{P}\left[\kappa_{\mathbf{x}^{\prime}}\right]$ $\geq T$ will decrease. This effect is shown in Figure 1, in which increasing the value of $T$ causes fewer data to lie above the threshold.

This suggests that we could use such a threshold $T$ to estimate the clinical labels for "abnormal" data; i.e., those from class $C_{2}$.

\subsection{Optimising a Classifier using a Threshold on the Multivariate Distribution}

In order to increase the separation between "normal" and "abnormal" data used for training a classifier, we can refine the $C_{1}$ and $C_{2}$ class labels provided by clinicians using the following rules:

i. Define the training set of "normal" data to be

$$
\left\{\mathbf{x} \in C_{1} \mid \mathrm{P}\left[\kappa_{\mathbf{x}}\right]<T\right\}
$$

ii. Define the training set of "abnormal" data to be

$$
\left\{\mathbf{x} \in C_{2} \mid \mathrm{P}\left[\kappa_{\mathbf{x}}\right] \geq T\right\}
$$

In order to examine the effect of varying values of the threshold $T, 75 \%$ of the data from $C_{2}$ that obeyed the above selection criterion were drawn at random, and an equal number of data from class $C_{1}$ were drawn at random. All remaining data from class $C_{2}$ were used as test data, and an equal number of data randomly selected from the unused data from class $C_{1}$ were used as test data.

The procedure involving threshold $T$ was used to process the training data only, and thus results obtained using the test data are independent of $T$, giving an accurate representation of the system's performance when classifying previously-unseen data. This is required in order to allow a fair comparison with classification performance obtained without using the proposed method.

Figure 2 shows the misclassification rates obtained when non-linear classifiers (multi-layer perceptron with a single layer of hidden units, or MLP, in this example) were compared over $N=50$ experiments. We note that the results are shown based on the test data, and that the classifier architecture was selected using 10-fold cross-validation.

It may be seen from the figure that, as the value of the threshold $T$ is increased from 0 to 1 , the number of false-positive misclassifications decreases while the number of false-negative misclassifications increases. This is because the "normal" training data (the refined version of class $C_{1}$ ) cover a larger locus as $T$ increases. Conversely, the "abnormal" training data (the refined version of class $C_{2}$ ) cover a smaller locus as $T$ increases. Thus, the resulting decision boundary of the classifier becomes less sensitive to abnormality with increasing $T$, because the classifier is trained with an increasingly large cluster of "normal" data and a decreasingly small set of clusters of "abnormal" data.

The figure shows that for $T \approx 0.4$, these misclassifications are minimised, which represents the "optimal" value of the threshold $T$ for processing the training data obtained from this data-set.

Similar results were obtained when applying the proposed technique with a support vector machine (SVM) classifier. Figure 3 shows the decision boundary obtained using an SVM both using the original $C_{1}$ and $C_{2}$ labels, and the "refined" labels created by using the procedure described previously. It may be seen that the SVM decision boundary obtained without application of the proposed method covers large areas of data space that correspond to physiological deterioration; e.g., the region that corresponds to tachypnoea (at BR $>30 \mathrm{rpm}$ ) for $\mathrm{HR}$ $\approx 75 \mathrm{bpm}$. In comparison, the SVM decision boundary obtained after application of the proposed method more closely describes the locus of "normal" data, in the centre of the data space, and accurately separates the four modes of data-space that correspond to apnoea, tachypnoea, bradycardia, and tachycardia. 


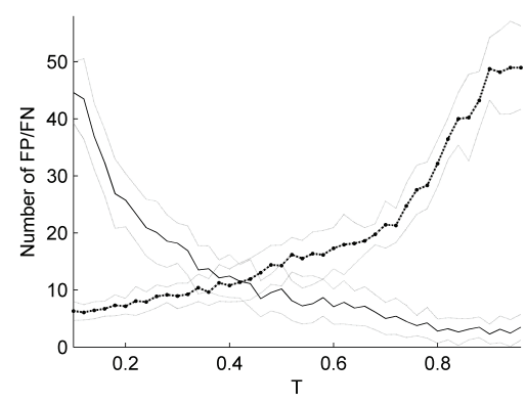

Figure 2: Misclassification performance evaluated using the test set, as threshold $T$ is varied. Mean false-positive (FP, shown by a solid line) and false-negative (FN, shown by a dotted line) errors over a range of $N=50$ experiments for each value of $T$, with a confidence interval on each value shown at \pm 1 standard deviation from the mean.
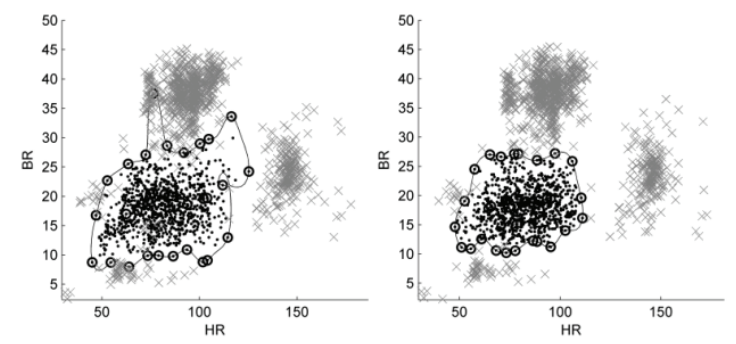

Figure 3: Using original (left-hand plot) and refined (righthand plot) $C_{1}$ and $C_{2}$ labels to construct a SVM classifier. The SVM produces a decision boundary shown by the black line, with support vectors indicated by circles.

\section{DISCUSSION AND FUTURE WORK}

We have presented a method of (i) estimating clinical labels, and (ii) using the technique to refine existing labels such that a classifier may be trained that better separates "normal" data from "abnormal" data, when compared with classifiers that do not use the proposed technique.

While this paper has presented the results of a bivariate analysis, such that the decision boundaries of classifiers may be examined and compared, the procedure should be applied in the dimensionality of the original data space; in the example considered by this paper, the data-set is 4-dimensional (HR, BR, $\mathrm{SpO}_{2}$, and SDA). The thresholding procedure is performed using the high-dimensional pdf, and so the proposed method of estimating and refining clinical labels is equally applicable to optimising classifiers in the original high-dimensional dataspace.

A further advantage of the proposed method is that, as described in Section 2, as the value of the threshold $T$ is increased, the distribution of data with probability $\mathrm{P}$ exceeding that threshold tends towards the distribution of data labelled as being class $C_{2}$ by clinicians. While there is no equal substitute for the annotations of clinical experts, it is impractical for a panel of experts to review 18,000 hours of continuous data. As described in Section 2, the $C_{2}$ labels were obtained as being a subset of those periods that exceeded univariate MET criteria for periods of at least four minutes, and so even these are not "gold standard" labels of the entire data-set. However, being able to estimate such labels is useful: the procedure can be applied to further, unlabelled data-sets in order to estimate their class $C_{2}$ labels. Thus, it may be possible to obtain automatically labelled data-sets from large unlabelled data-sets, which would previously require the use of an unsupervised classification approach, such as the one-class method described in Section 1, and in (Tarassenko, 2005) and (Tarassenko, 2009).

\section{REFERENCES}

Buist, M. D., Jarmolowski, E., Burton, P. R., Bernard, S. A., Waxman, B. P., Anderson, J., 1999: Recognising Clinical Instability in Hospital Patients Before Cardiac Arrest or Unplanned Admission to Intensive Care. Med. J. Australia, Vol. 171 8-9.

NPSA, 2007. National Patient Safety Association: Safer Care for Acutely Ill Patients: Learning from Serious Accidents. Tech. Rep

Tarassenko, L., Hann, A., Patterson, A., Braithwaite, E., Davidson, K., Barber, V., Young, D., 2005: Multiparameter Monitoring for Early Warning of Patient Deterioration. Proc. 3rd IEE Int. Seminar on Medical Applications of Signal Processing, London, 71-6.

Smith, G. B., Prytherch, D. R., Schmidt, P. E., Featherstone, P. I.,2008: Review and Performance Evaluation of Aggregate Weight “Track and Trigger" Systems. J. Resuscitation, Vol. 77, 170-179.

Tarassenko, L., Clifton, D. A., Bannister, P. R., King, S., King, D., 2009: Novelty Detection. In: Staszewski, W. J., Worden, K. (eds), Encyclopaedia of Structural Health Monitoring, Wiley.

Bishop, C. M., 2006.: Pattern Recognition and Machine Learning. Springer-Verlag. 